\title{
A graphic code, one hundred architectural projects
}

\section{SIGRADI2018 TECHNOPOLITICAS \\ xxii congresso da sociedade iberoamericana de gráfica digital 22th conference of the iberoamerican society of digital graphics 07|08|09|novembro|2018 iau usp | são carlos | sp br}

\author{
Alejandro Folga \\ FADU | Uruguay | alfotocopias@gmail.com \\ Fernando García Amen \\ FADU | Uruguay | efe@fadu.edu.uy
}

\begin{abstract}
This paper presents the results of the development of a graphic coding implementation system that was created to produce a series of diagrams integrated into a pre-existing digital guide platform (www.plexo.edu.uy). The aim of this coding system is - among others - to present a selection of one hundred architectural projects from different parts of the world, so that it serves as a guide in an academic trip made by architecture students. The drawings were made by advanced students who were taking an undergraduate course.
\end{abstract}

Keywords: Research-teaching; Architecture; Graphic Coding

\section{INTRODUCCIÓN}

Este paper presenta el desarrollo e implementación de un sistema de codificación gráfica que fue diseñado específicamente para producir diagramas de una selección de cien obras arquitectónicas de diferentes lugares del mundo. El dibujo de los diagramas se llevó a cabo mediante un ejercicio pedagógico realizado con estudiantes avanzados que cursaban una asignatura electiva de la carrera de Arquitectura. Esta asignatura se realizó en el marco del Proyecto Académico "Plexo Territorios lúdicos", rector del Viaje de Arquitectura de 2018. El resultado del ejercicio se integró en una plataforma digital preexistente (www.plexo.edu.uy) que sirve de guía en el viaje académico realizado por los estudiantes de la Facultad de Arquitectura, Diseño y Urbanismo (FADU) de la Universidad de la República (UdelaR) en Montevideo, Uruguay.

Otra manera de presentar nuestro trabajo es decir que su objetivo general fue desarrollar un ejercicio pedagógico con estudiantes de arquitectura que realizarían un viaje académico. Mediante este ejercicio se buscó trascender la mera repetición de los dibujos originales, promoviendo el desarrollo de lecturas conceptuales (analíticas o sintéticas) de cada caso estudiado. Entendidos de esta manera, los gráficos suponen un nuevo aporte a la actividad académica del Viaje, ya que proponen miradas proyectuales o lecturas explicativas de los ejemplos representados. En ese sentido, podemos decir que el objetivo particular del trabajo fue la construcción de un código gráfico propio, capaz de reinterpretar y valorar obras arquitectónicas que posteriormente serían visitadas por los estudiantes que hicieron los dibujos.

Como antecedentes de este proyecto de enseñanzainvestigación debemos mencionar el trabajo realizado por Plexo (Amén, Álvarez, Portillo y Buzó, 2015) y la APP Nómada ${ }^{1}$, creada por estudiantes de arquitectura (Mora, 2016).

\section{METODOLOGÍA}

La metodología que implementamos se desarrolló en tres partes. La primera parte implicó realizar una investigación previa sobre los códigos gráficos utilizados en distintas publicaciones de arquitectura. La segunda parte requirió la definición de un protocolo de dibujo mediante una serie de pautas y criterios comunes. La tercera parte consistió en desarrollar un ejercicio con los estudiantes encargados del redibujo digital (formato CAD).

\section{INVESTIGACIÓN PREVIA}

El objetivo de la primera parte del trabajo fue reflexionar sobre los conceptos gráficos a partir de los cuales se realizaría el redibujo de las cien obras elegidas. Esto implicó que el equipo docente llevase a cabo una breve investigación exploratoria ${ }^{2}$ sobre diferentes publicaciones y webs dedicadas a recopilar, presentar o estudiar gráficamente obras de arquitectura: guías, manuales y trabajos analíticos sobre proyectos.

De todos los casos estudiados los más interesantes son aquellos que no se plantearon la mera repetición de los dibujos originales, sino que se proponían trascenderlos o re-interpretarlos. Para lograr estos objetivos dichas publicaciones incorporan recursos gráficos que permiten el análisis o la explicación proyectual de los edificios.

A partir de la revisión bibliográfica destacamos tres publicaciones ${ }^{3}$ que resultaron los principales referentes para el desarrollo de nuestro propio código gráfico:

- French (2008). Vivienda colectiva paradigmática del siglo $X X$.

- Fernández Per, Mozas y Ollero. (2013). 10 historias sobre vivienda colectiva: Análisis gráfico de diez obras esenciales.

- Fernández Per, Mozas y Arpa (2009). HoCo: Density, Housing, Construction \& Costs 
Los tres casos utilizan el dibujo técnico para estudiar ejemplos de vivienda colectiva. Uno de los recursos que estas publicaciones tienen en común es el uso de áreas con relleno de color para señalar algunos sectores en las plantas o en los cortes. Este recurso permite analizar la organización programática de los edificios, destacando los espacios colectivos con respecto a los privados (ver ejemplos de estos libros en las figuras 1,2 y 3 ).
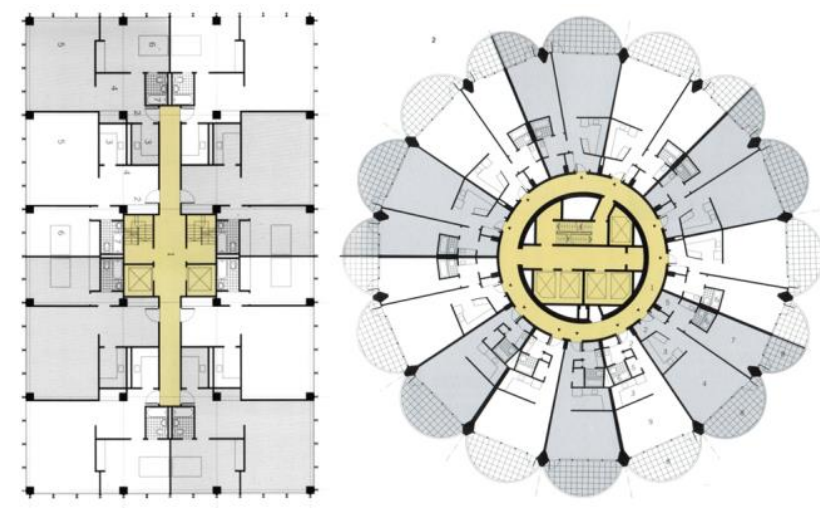

Figura 1: Vivienda colectiva paradigmática del siglo $X X$. French, Hilary (2008)
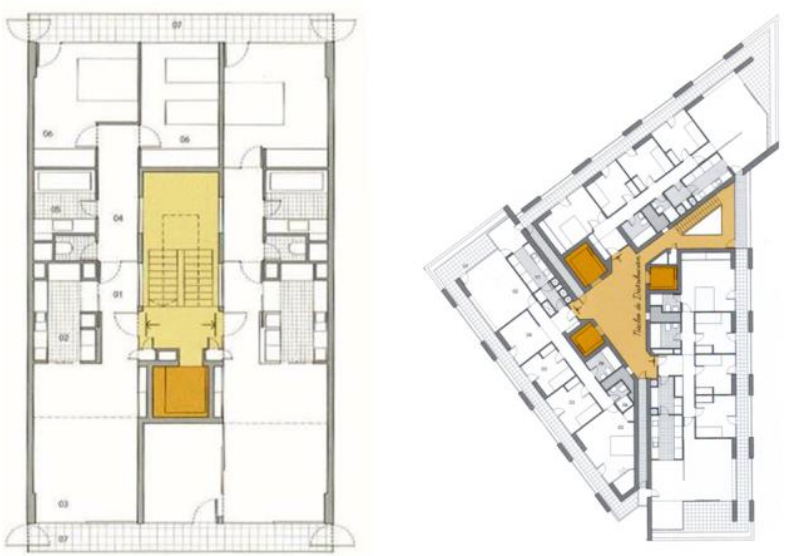

Figura 2: 10 historias sobre vivienda colectiva: Análisis gráfico de diez obras esenciales. Fernández Per, Mozas, y Ollero (2013)
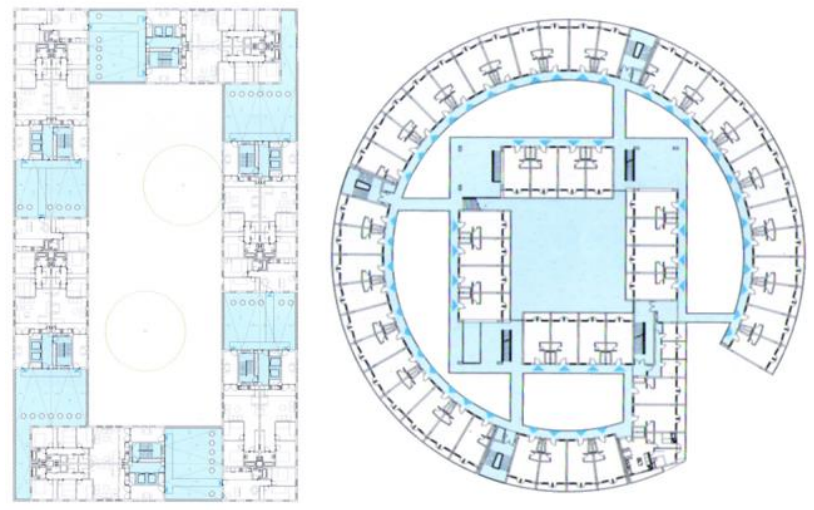

Figura 3: HoCo: Density, Housing, Construction \& Costs, Fernández Per, Mozas y Arpa (2009)
Claramente, se trata de un criterio gráfico-analítico que depende de las ideas que se desean comunicar. En este sentido, es conveniente mencionar aquí la justificación del dibujo analítico que Elena Mata Botella (2002) incluye en la introducción de su tesis de doctorado.

Cuando se analiza gráficamente, es cuando se hace patente la capacidad del dibujo para ser selectivo frente a una realidad mucho más compleja. Es por esta capacidad de selección del dibujo que nos permite acercarnos más a la esencia que a la apariencia de los objetos (El subrayado es del original) (p.7).

Alineados con estos objetivos, en lugar de repetir los dibujos preexistentes nos propusimos generar nuevos gráficos. Asumimos que una forma de producir esta innovación era mediante diagramas ${ }^{4}$ a partir de los cuales pudiésemos plantear una mirada analítica sobre los ejemplos, a la vez que realizábamos una sistematización de las obras estudiadas.

\section{HACIA UN NUEVO CÓDIGO}

En el libro Código Gráfico (Pantaleón et al., 2011, p.12) se establece la importancia que la codificación gráfica tiene para la comunicación de proyectos:

Si partimos del concepto de código - "sistemas de signos y de reglas que permiten formular y comprender un mensaje" - y abordamos las definiciones de signo - "objeto, fenómeno o acción material que natural o convenientemente representa y sustituye a otro objeto, fenómeno o señal" — y de regla - "lo que se debe obedecer - seguir por estar así establecido"comprenderemos la necesidad por parte del dibujante-arquitecto-comunicador de conocer y manejar un código gráfico.

Por lo tanto, para generar un código, definir sus signos y explicar sus reglas, antes debemos establecer qué es lo que queremos comunicar. En ese sentido, luego de hacer un primer análisis de los casos que se iban a dibujar, tomamos una serie de decisiones metodológicas para luego definir el tipo de diagramas que produciríamos.

A diferencia de las publicaciones usadas como referentes -que tratan sobre vivienda colectiva moderna y contemporánea- las obras que nosotros debíamos dibujar abarcan un amplio abanico de programas y tipos arquitectónicos, además de que pertenecen a diferentes momentos históricos. Por ello, partimos de problematizar ${ }^{5}$ la codificación. Entendimos que en lugar de generar un código cerrado era conveniente plantearlo como un código abierto. Es decir, al inicio del trabajo nuestra codificación no estaba totalmente definida sino que solo se planteaban algunas pautas gráficas básicas.

Las decisiones tomadas en esta etapa nos permitieron generar una primera versión del código gráfico, que posteriormente fue presentada a los estudiantes y puesta a prueba mediante el ejercicio. En esa instancia nuestro código sería sometido a un primer ajuste para adaptarse mejor a los casos estudiados. 

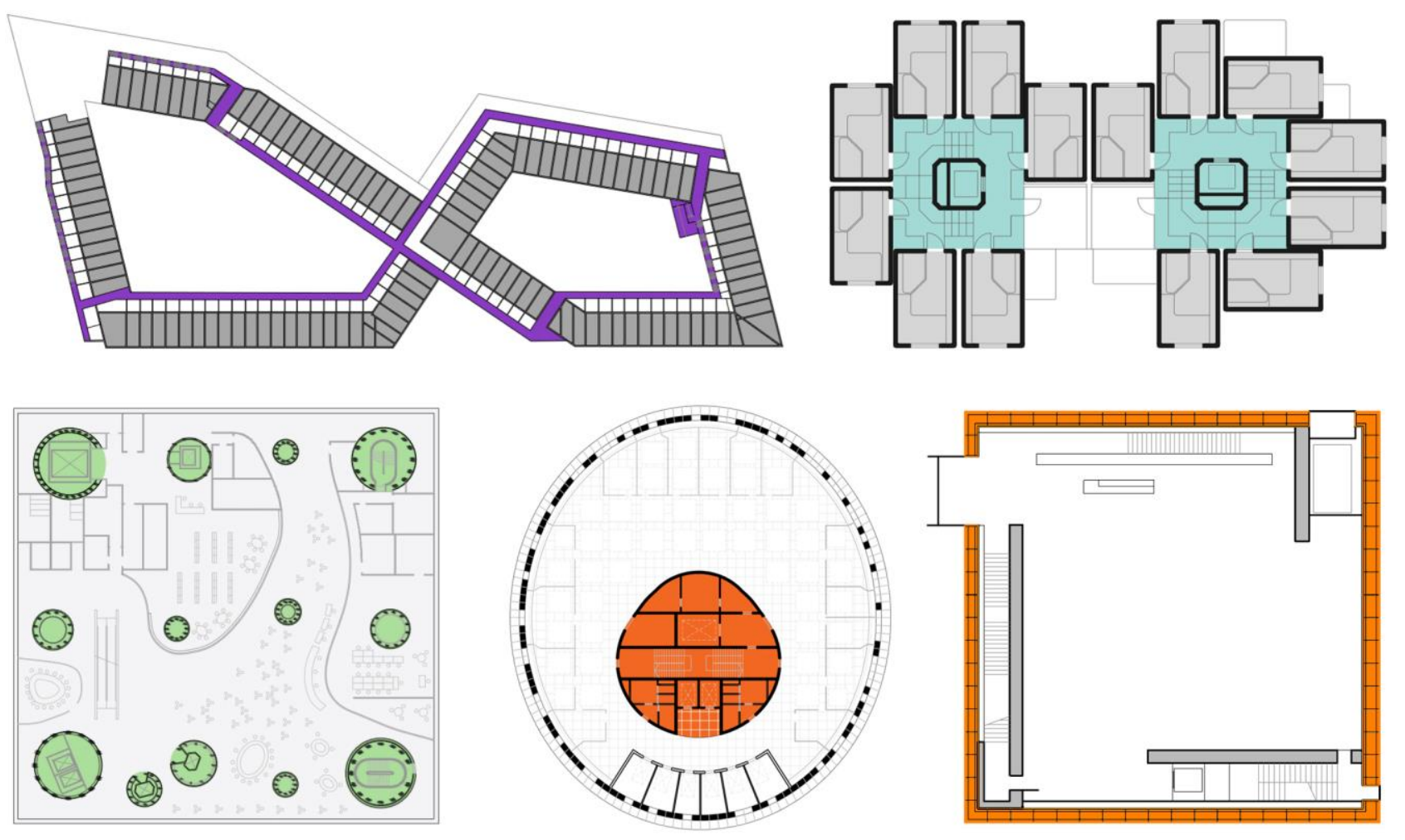

Figura 4: Plantas de diferentes edificios. Realizados por los estudiantes del curso.

\section{Síntesis de los gráficos}

Dado que el trabajo estaba destinado a una plataforma diseñada para teléfonos inteligentes, nuestros gráficos debían adecuarse al reducido tamaño de pantalla de estos dispositivos. Acorde a esa condicionante, el nivel admisible de detalles debía atenuarse y simplificarse. Por ello nos impusimos una restricción, realizar solamente geometrales en diédrico (plantas, cortes y fachadas) evitando así las axonometrías y las perspectivas. De esta forma se simplificaba el trabajo a la vez que se obtenían dibujos de una mayor homogeneidad gráfica.

Por otro lado, era preferible que los estudiantes dibujasen cortes y plantas ${ }^{6}$, pues son piezas que permiten un mayor grado de abstracción con respecto a las fachadas. Además, por ser más conceptuales expresan mejor las relaciones entre diferentes espacios, y por lo tanto resultan más adecuadas para generar diagramas.

\section{Usos del color}

Para conseguir mayor unidad entre los diferentes gráficos definimos un reducido espectro cromático que consistió en no usar más de dos colores por dibujo. A su vez, esa limitada paleta debía ser compatible con las pautas gráficas de la plataforma digital preexistente. Esto determinó que en cada gráfico se utilicen colores que se correspondan con los cuatro bloques en que se divide el recorrido del viaje académico ${ }^{7}$.

Las condicionantes anteriores llevaron a definir tres reglas que los dibujantes debían cumplir. La primera regla planteaba que en cada dibujo solo podían utilizarse los colores que caracterizaban al bloque de viaje al que pertenecía la obra. La segunda regla era que los colores que caracterizan a cada bloque se podían aplicar con dos diferentes grados de saturación: pleno $(100 \%$ de opacidad) y medio (60\% de opacidad). La tercera regla permitía, además de los dos colores por bloque, dos valores de gris (gris 254 y gris 253; en el código ASCII correspondiente a AutoCAD) que eran comunes a todos los bloques del viaje. En resumen, a partir de estas reglas cada estudiante podía utilizar ambas variantes del color característico del bloque, o tenía la alternativa de usar uno de los colores del bloque y uno de los grises.

\section{EJERCICIO CON ESTUDIANTES}

La última parte del trabajo consistió en el redibujo digital, mediante la técnica de "calcado" de gráficos existentes en programas CAD, de una serie de 100 diferentes obras de arquitectura. Para ello desarrollamos un ejercicio didáctico con estudiantes que cursaban una asignatura electiva que formó parte de la curricularización ${ }^{8}$ del viaje académico y surgió como propuesta del Equipo Director ${ }^{9}$ encargado de la organización del viaje. Por lo tanto, el grupo de estudiantes que realizó el ejercicio estaba directamente relacionado con las guías de viaje y podíamos suponer que también tenía un genuino interés en realizar esta actividad.

Nuestro mayor desafío fue organizar a un amplio grupo de estudiantes para dibujar un más amplio conjunto de edificios, respetando un código gráfico común a todos los casos. Esta condicionante implicó la definición de un protocolo de uso de herramientas digitales (templates, formatos de entrega, manejo de archivos, etc.).

\section{OBJETIVOS PEDAGÓGICOS DEL EJERCICIO}

En el artículo "Representación técnica" Inmaculada López Vílchez (2011) plantea tres funciones básicas para las que una representación puede servir: "para conocer, para ver y mostrar, para producir y fabricar” (p. 158). 

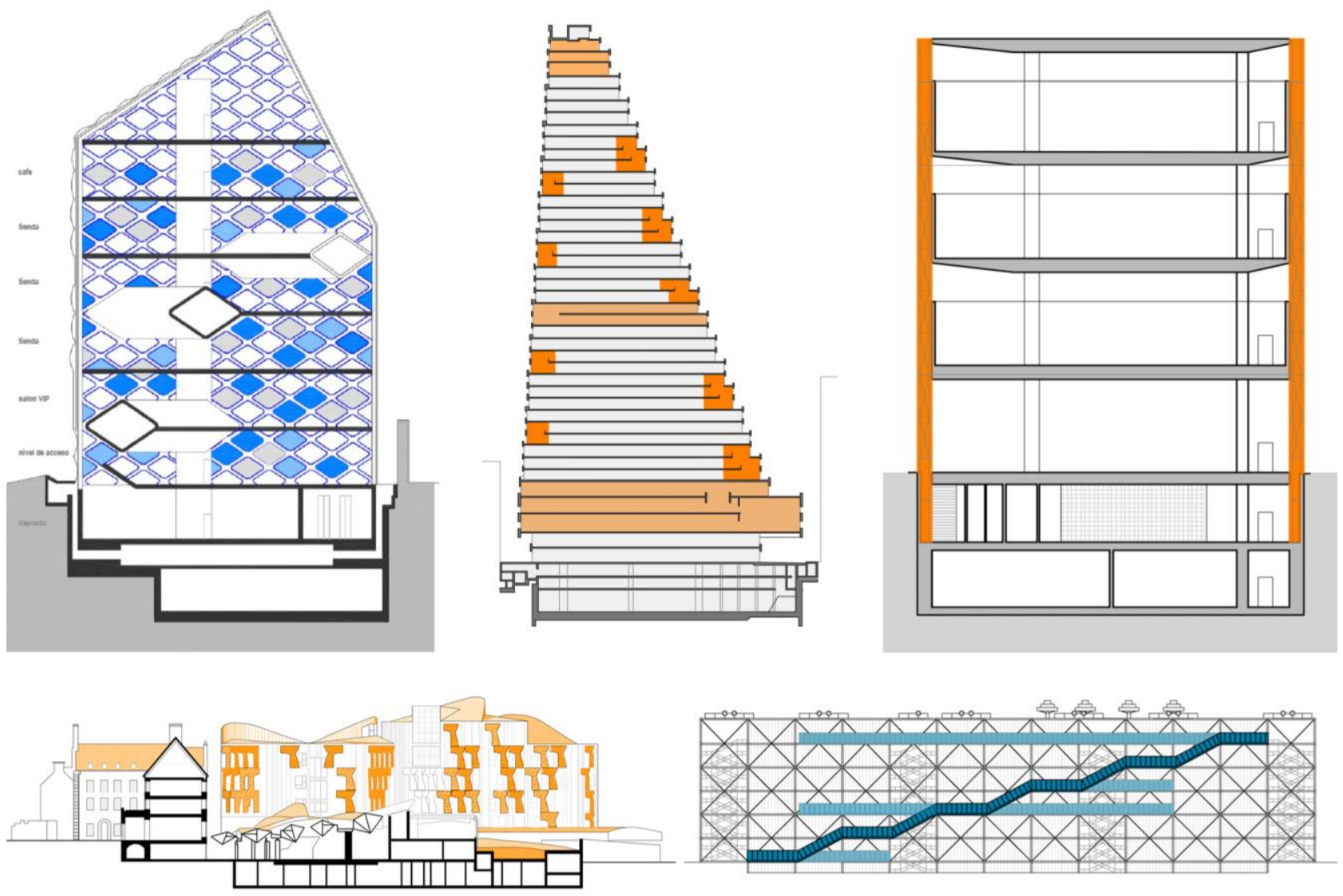

Figura 5: Cortes y fachadas de diferentes edificios. Realizados por estudiantes del curso.

En nuestro caso resultan pertinentes la primera y la segunda función. En ese sentido, podemos definir tres objetivos que orientaron el ejercicio académico:

- Propender a que los estudiantes comprendan mejor las obras que luego visitarán. Porque, redibujar arquitectura es una forma de conocer lo dibujado, o de profundizar y acrecentar el conocimiento que ya se posee.

- Presentar los edificios de manera didáctica. Dado que los dibujos están destinados a una guía para estudiantes de arquitectura, nuestro trabajo se constituía en un aporte para todo el grupo de viaje.

- Proponer un posible recorrido de las obras y espacios. Como los dibujos se integrarían a una guía de viaje, resultaba válido pensar en ellos como un apoyo a la experiencia del visitante.

A partir de lo anterior se consolidó el requerimiento de plantear una lectura proyectual de los edificios, de manera que los gráficos enfaticen o destaquen algún aspecto notable de cada proyecto. Un listado, no exhaustivo, de posibles lecturas comprendería: análisis espaciales y compositivos, organización estructural, definición tipológica, vínculos contextuales, etc. En definitiva, cada dibujo debía pensarse como un diagrama cuya función era presentar las ideas de los proyectos.

Para cumplir con estos cometidos se instó a los estudiantes a elegir los dibujos que resultasen más representativos, es decir: aquellos que mejor comunican las ideas y atributos proyectuales de cada obra.

\section{ETAPAS DEL EJERCICIO}

\section{Etapa 1- Presentación}

El ejercicio comenzó con una clase teórica y colectiva en la que se presentaron los objetivos del trabajo, las pautas para realizar los gráficos y los diferentes formatos de entrega $^{10}$. En esa primera instancia asignamos ${ }^{11}$ los 100 edificios a los 51 estudiantes inscriptos en el curso. Esto implicó que cada estudiante debía realizar los gráficos de dos edificios diferentes. Luego de esta clase, como instancia previa a la tarea de dibujar, los estudiantes realizaron una búsqueda de información sobre los proyectos que les fueron asignados (textos, análisis, fotografías, y gráficos) para así conocerlos y entenderlos.

\section{Etapa 2- Seguimiento docente}

La segunda etapa implicó un proceso de sucesivos ajustes, para lo cual definimos una serie de instancias semanales de corrección. Para coordinar al numeroso grupo de estudiantes que participó del ejercicio contamos con un equipo docente multicátedra (docentes del EDD, provenientes de diferentes áreas académicas de la FADU) que se encargó de realizar el seguimiento de los estudiantes y controlar el avance de los dibujos.

\section{Etapa 3- Ajuste final e implementación}

Luego de que los estudiantes culminaran con el trabajo asignado, el equipo docente coordinador fue responsable de procesar todos los gráficos para poder incluirlos en las guías digitales. Esta tarea implicó realizar algunos ajustes mínimos en los dibujos entregados ${ }^{12}$. Por ejemplo: se normalizaron tamaños de texto, se agregaron símbolos de referencia (escala gráfica y símbolos de Norte) y se 
ajustaron los colores de relleno (sólo en aquellos casos que no se respetó el código establecido).

\section{RESULTADOS}

Finalmente, 27 estudiantes culminaron el ejercicio y se confeccionaron 54 dibujos en total. En las figuras 4 y 5 presentamos una selección de algunos de los gráficos que evaluamos como los más adecuados en función de los objetivos planteados. La figura 4 muestra gráficos de plantas (realizados, respectivaente, por los estudiantes Claudia Bonari, Clara Lagarriga, Paula Miller, Paula Miller y Mathias Cristiano) y la figura 5 muestra cortes y fachadas (realizadas por los estudiantes Ana Clara Sica, Sebastián Ibarra, Mathias Cristiano, Ignacio Fontán y Anifrid Fernández).

El producto final de este trabajo, es decir, la producción de geometrales interpretados y valorados para la comprensión funcional, concisa y veloz del observador, se integró a la plataforma general del Viaje en diferentes formatos: como parte del contenido de las guías interactivas de viaje, como insumos de la app Nómada, previendo geolocalización y posicionamiento, pero, sobre todo, como producto final de una actividad de reflexión y pensamiento sobre una serie de edificios icónicos, previamente a su visita.

\section{DISCUSIÓN}

Como evaluación general del trabajo consideramos que por tratarse de un proyecto que desarrolla enseñanza e investigación en paralelo la experiencia fue doblemente provechosa. En lo relativo a la investigación, el mayor aporte fue la definición del propio código gráfico y la producción de una serie de dibujos originales, específicamente generados para formar parte de las guías. En relación a la enseñanza, el principal aporte del trabajo consistió en desarrollar un ejercicio pedagógico al mismo tiempo que debíamos coordinar un numeroso grupo de dibujantes trabajando bajo una consigna común. En definitiva, se trata de una investigación sobre la representación gráfica y también una actividad didáctica, donde ambas partes están mutuamente imbricadas y se retroalimentan.

\section{EVALUACIÓN PEDAGÓGICA}

La evaluación primaria de la metodología pedagógica implementada nos sugiere que el ejercicio presentó una complejidad excesiva para algunos estudiantes. A pesar del buen nivel obtenido en la mayoría de los trabajos, no podemos desconocer que una proporción significativa de los inscriptos no logró completar el ejercicio. Por diferentes razones el $47 \%$ de los estudiantes abandonó el curso, lo que nos lleva a cuestionar la didáctica aplicada.

Por otro lado, debemos reconocer que algunos de los gráficos que sí fueron entregados no aportan una mirada significativa del caso estudiado, e incluso pueden llegar a mostrar una lectura confusa o engañosa. A pesar del significativo empeño puesto por los estudiantes $-\mathrm{y}$ del correspondiente apoyo que estos recibieron por parte del equipo docente - no todos los edificios consiguen buenos resultados al hacerlos pasar por el tamiz de la codificación.
Consideramos que uno de los principales factores causantes de dicho problema fue el escaso tiempo que disponíamos para realizar el trabajo. Aunque no podemos obviar que este conflicto también puede estar relacionado con la exigencia de incluir por lo menos un color para cumplir con la codificación. Esta obligación pudo llevar a algunos estudiantes a producir dibujos en los que el uso del color resulta un recurso forzado. Por ello, una de las conclusiones que extraemos de esta experiencia es que los recursos gráficos no siempre resultan pertinentes ni tienen validez universal.

Por todo ello, consideramos que el sistema de codificación requiere de algunos ajustes. Cuando el viaje académico haya culminado (diciembre de 2018) estaremos en condiciones de hacer una evaluación del desempeño de la plataforma. Los resultados definitivos sobre su aprovechamiento podrán ser mensurados cuando se tengan registros de uso, niveles de satisfacción y feedback general de los estudiantes a partir del uso de esta herramienta. Para esa instancia nos hemos propuesto realizar una revisión completa del código y plantear adaptaciones de cara a las posibles ediciones futuras de las guías.

A pesar de todos los inconvenientes antes mencionados, debemos recalcar la importancia de este tipo de actividades, pues se trata de un trabajo que explota algunas de las potencialidades inherentes de este particular viaje de estudios.

Sin dudas, el mayor aporte del trabajo fue para aquellos que cursaron la asignatura electiva, dado que el ejercicio de generación de diagramas fortalece los criterios de análisis y facilita el abordaje de la arquitectura como objeto de estudio. En definitiva, el ejercicio individual realizado por cada estudiante complementa su preparación para el viaje académico. No obstante, además de contribuir a la formación de aquellos estudiantes que cursaron la asignatura, el objetivo más ambicioso de este trabajo fue producir un resultado que trasciende a los participantes del curso y se colectiviza a todo el grupo de estudiantes que viaja. Por estas dos vías nuestro trabajo permite una mejor preparación del Viaje como evento académico y experiencia fundamental para el conocimiento de la arquitectura.

\section{DOCENCIA E INVESTIGACIÓN}

Con referencia a la relación entre enseñanza e investigación, el profesor Porfirio Morán Oviedo (2003) reclama un docente doblemente formado: en la disciplina y también en pedagogía. Este perfil lo encuentra en aquellos que denomina docentes-investigadores, pues son quienes "enseñan lo que investigan y hacen de su práctica docente objeto de estudio" (p. 18).

En ese sentido $\rightarrow$ y volviendo a lo planteado en la Introducción - entendemos necesario defender esta modalidad de trabajo en la que se produce una clara retroalimentación entre enseñanza e investigación universitarias. En definitiva, nuestra investigación estuvo pensada para generar una herramienta didáctica que pretende una mejora de la enseñanza y, por otro lado, el ejercicio pedagógico se integró como herramienta en la realización de una investigación sobre codificación gráfica $^{13}$.
5 


\section{DOCENCIA Y EXTENSIÓN}

Consideramos que la principal contribución metodológica de este trabajo fue la definición de un protocolo de dibujo que, en una instancia posterior, puede extrapolarse a un mayor universo de estudio, e incluso podría ser aplicado en otras investigaciones pedagógicas ${ }^{14}$.

Por último, en la medida que la plataforma digital puede ponerse a disposición de otros interesados (incluso por fuera de los grupos de viaje) se trata también de un proyecto de extensión universitaria. De esta manera nuestra experiencia completa el círculo virtuoso de la integralidad universitaria (Kaplún, 2012). Esto nos vincula con las tecnopolíticas - tema central del SIGRADI XX々 en tanto la tecnología de las guías digitales permite un crecimiento de la información y del conocimiento sobre arquitectura.

\section{NOTAS}

1. Sobre la APP Nómada puede consultarse la nota: "Estudiantes de arquitectura uruguayos crean guía de viaje para smartphones" (2016).

2. Sobre las investigaciones exploratorias Hernández Sampieri, Fernández Collado y Baptista (1997) nos dicen que estas se efectúan "cuando el objetivo es examinar un tema o problema de investigación poco estudiado o que no ha sido abordado antes" (p. 13). En ese sentido, Dankhe (1986) establece que los resultados de los estudios exploratorios "en pocas ocasiones constituyen un fin en sí mismos, por lo general determinan tendencias, identifican relaciones potenciales entre variables y establecen el 'tono' de investigaciones posteriores más rigurosas" Las cursivas son del autor. (Citado en Hernández et al., 1997, p.13). En ese sentido, nuestro trabajo cumple con las pautas para definirlo como investigación exploratoria.

3. También debemos mencionar el libro de Peter Eisenman (2010) Diez edificios canónicos. Este libro aportó una pauta gráfica definitiva para nuestro trabajo. Se trata de dibujos que incorporan un solo color para analizar proyectos. Pero los dibujos de esa publicación tienen un carácter muy diferente al trabajo que nosotros proponíamos, por eso nos referiremos principalmente a los tres libros sobre vivienda.

4. Josep María Montaner (2014) opina que los diagramas contemporáneos "tienen sus raíces en la voluntad humana de sistematizar y objetivar, en una aspiración a la abstracción que busca una certeza fiable de partida" (p. 20). Además, distingue claramente entre los diagramas que sirven para representar una "lectura analítica de la realidad" y los que "sirven para proponer y proyectar" (p. 10). En este trabajo utilizamos la primera de las interpretaciones.

5. Por problematizar se entiende a una acción que consiste en poner en cuestión un tema determinado para poder analizar y discutir los aspectos que plantean mayor complejidad.

6. Aunque sí se dibujaron algunas fachadas, por lo general consideramos que lo que aporta una fachada es más fácil de sustituir por una fotografía.

7. Tomando como referencia el código ASCII, utilizado por el software AutoCAD, los colores de saturación plena que fueron adoptados para cada bloque son: Bloque 1, color 94; Bloque 2, color 140; Bloque 3, color 200 y Bloque 4, color 30 .

8. La curricularización del viaje de estudios (entendido como una experiencia fundamental para los estudiantes de arquitectura) implica complementarlo con asignaturas electivas, que permiten a los estudiantes obtener créditos académicos a la vez que se generan productos concretos que aportarán conocimientos a su propia experiencia en el viaje.

9. Según el reglamento del Grupo de Viaje de Arquitectura, cada año se realiza una convocatoria en la que se postulan diferentes equipos docentes de la FADU. Uno de estos es elegido como Equipo Docente Director (EDD). En 2018 el EDD estuvo compuesto por: Raúl Buzó, Ángel Armagno, Fernando García Amen, Gabriela Barber, Pablo Canén, Alberto De Austria, Jimena Abraham, Serrana Robledo, Laura Vizconde, Roberto Langwagen, Juan Pablo Portillo y Marcelo Payssé.

10. Los formatos de entrega fueron tres: una versión impresa y dos archivos (DWG y PDF).

11. Por lo general es preferible que los estudiantes elijan los casos para conseguir un mayor compromiso con el trabajo. No obstante, nosotros optamos por repartirlos de manera aleatoria ya que disponíamos de poco tiempo y las obras eran numerosas.

12. En esta tarea participó activamente Ana María López.

13. También podríamos invertir el orden de estas dos oraciones coordinadas y el resultado seguiría siendo válido.

14. Estamos pensando en diseñar un ejercicio didáctico (con estudiantes avanzados de cursos de proyecto de arquitectura) en el que ensayen la aplicación de una codificación específica en diversas escalas de edificios o proyectos.

\section{REFERENCIAS}

Amen, Fernando García; Álvarez, Marcelo Payssé; Portillo, Juan Pablo; Buzó, Raúl Felipe; "Plexo. Una travesía multisensorial", p. 493-496 . In: . São Paulo: Blucher, 2015. ISSN 2318-6968, ISBN: 978-85-8039-136-7

Fernández Per, Aurora; Mozas, Javier y Arpa, Javier. (2009) HoCo: Density, Housing, Construction \& Costs. Victoria Gasteiz: a+t ediciones.

Fernández Per, Aurora; Mozas, Javier y Ollero, Alex. (2013). 10 historias sobre vivienda colectiva: Análisis gráfico de diez obras esenciales. Victoria Gasteiz: a+t ediciones.

French, Hilary. (2008). Vivienda colectiva paradigmática del siglo $X X$. Barcelona: Gustavo Gili.

Kaplún, Gabriel (2012). La integralidad como movimiento instituyente en la universidad. En: InterCambios, no 1, 2012. Disponible en: http://intercambios.cse.edu.uy.

López Vílchez, Inmaculada. (2011). "Representación técnica”. En L. Cabezas. (Coord.) Dibujo y construcciòn de la realidad. (pp. 153-196). Madrid: Cátedra.

Montaner, Josep María. (2014). Del diagrama a las experiencias, hacia una arquitectura de la acción. Barcelona: G. Gili.

Mata Botella, Elena. (2002). Tesis de doctorado inédita, El análisis gráfico de la casa, UPM, Madrid.

Mora, Pola (2016). "Estudiantes de arquitectura uruguayos crean guía de viaje para smartphones". Recuperado de: https://www.plataformaarquitectura.cl.

Morán Oviedo, Porfirio. (2003). El reto pedagógico de vincular la docencia y la investigación en el espacio del aula. Revista de Contaduría y Administración, (211), pp. 17-30.

Pantaleón, Carlos; Fernandez, Laura, Folga, Aleandro; Garat, Daniela y Parodi, Aníbal. (2011) Código Gráfico. Montevideo: Ediciones Udelar.

Sampieri, Roberto; Fernández Collado, Carlos y Baptista Lucio, Pilar. (1997). Metodología de la Investigación. México: Mc Graw Hill. 